\title{
Intrinsic Fluorescence Spectra of Tryptophan, Tyrosine and
}

\section{Phenyloalanine}

\author{
YANG Hui ${ }^{1, a^{*}}$, XIAO Xue ${ }^{2, b}$, ZHAO Xuesong ${ }^{2, c}$ and WU Yan ${ }^{1, d}$ \\ 1. New Star Application Technology Institute, Hefei, Anhui 230031, China \\ 2. Key Lab. of Environmental Optics \& Technology, AIOFM, CAS, Hefei, Anhui 230031, China

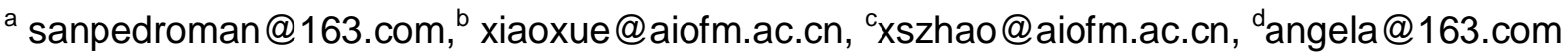

Key words: Fluorescence spectra, tryptophan, tyrosine, phenyloalanine

Abstract: This paper presents the intrinsic fluorescence spectra characteristics of tryptophan, tyrosine and phenyloalanine measured with 3D fluorescence spectrophotometer. Two strong fluorescence peaks of tryptophan locate at $\lambda \mathrm{ex} / \lambda \mathrm{em}=230 / 348 \mathrm{~nm}$ and $280 / 348 \mathrm{~nm}$, three strong fluorescence peaks of tyrosine locate at $\lambda \mathrm{ex} / \lambda \mathrm{em}=202 / 304 \mathrm{~nm}, \lambda \mathrm{ex} / \lambda \mathrm{em}=220 / 304 \mathrm{~nm}$ and $275 / 304 \mathrm{~nm}$, and two fluorescence peaks of phenyloalanine locate at $\lambda \mathrm{ex} / \lambda \mathrm{em}=210 / 280 \mathrm{~nm}$ and $\lambda$ ex $/ \lambda \mathrm{em}=260 / 280 \mathrm{~nm}$ were found. The linear relationship of fluorescence intensity to solution concentration were also observed in condition of low solution concentration. The influence of $\mathrm{pH}$ of solution to the fluorescence intensity was also measured and discussed.

\section{Introduction}

Of the twenty naturally occurring amino acids that make all proteins, three are conjugated with aromatic ring side chains, and therefore intrinsically optically active: tryptophan (Trp), tyrosine (Tyr) and phenyloalanine (Phe). Tryptophan is excited at wavelengths around $280 \mathrm{~nm}$ and emits fluorescence in a peak from 300-400 nm. NADH is excited between 270 and $400 \mathrm{~nm}$ and emits between 400 and $600 \mathrm{~nm}$, and Riboflavin is excited at 300-500 nm and emits mostly between 400 and $700 \mathrm{~nm}^{[1,2,3]}$. The fluorescence from a complex mixture of proteins mainly originates from $\operatorname{Trp}$ residues. The indole group of tryptophan residues is the dominant source of absorbance and emission in protein. And the excitation and emission bands of these bio-fluorophores are quite overlapped within the EEM area whose excitation wavelength/emission wavelength scope is $230-270 \mathrm{~nm} / 260-340 \mathrm{~nm}$. So through the detection of intrinsic fluorescence characteristics of tryptophan, tyrosine and phenyloalanine, the comparative contents of various kinds of organic components can be analyzed and resolved, and finally the classification of bio-aerosol/bio-agent can be reached.

In this paper, the intrinsic 3D fluorescence spectra of tryptophan, tyrosine and phenyloalanine were obtained with an F-7000 FL spectrophotometer and the individual excitation/emission spectra were decomposed successfully from the mixed solutions and simulated mixtures.

\section{Experimental Section}

\section{Instruments and Reagents}

The Molecular $\Sigma \mathrm{H} 2 \mathrm{O}$ ultra pure water machine (Shanghai Molecular Co. Ltd) was used to generate the ultra purified water, UPW whose $\mathrm{pH}$ value is 5.4. L-Tryptophan mother liquid were compounded with L-Tryptophan from Sigma co.Ltd whose minimum purity equals to $99 \%$, the L-Tyrosine mother liquid were compounded with L-Tyrosine from Sigma co.Ltd whose minimum purity equals to $98 \%$, and the L-Phenylalanine mother liquid were compounded with L- 
Phenylalanine from USB co.Ltd whose minimum purity equals to $99 \%$. All reagents and materials were weighed with Mettler Toledo precise electronic balance, and dissolved with $\mathrm{KH}_{2} \mathrm{PO}_{4}$ buffer or $\mathrm{Na}_{2} \mathrm{HPO}_{4}$ buffer with different $\mathrm{pH}$ values of 6.5, 6.6, 6.8, 7.0, 7.2, 7.3, 7.4, 7.6 and 8.0.

The L-Tryptophan, L-Tyrosine and L-Phenylalanine mother liquid concentration are 20, 21 and $40 \mathrm{mg} / \mathrm{L}$ respectively. The all mother reagent solutions were transferred through DragonLab whole disinfection manual single channel adjustable liquid shifter and dilute to working solutions of different concentrations. All reagents were of analytical grade, all solutions and put in amber glass bottles and stored in a refrigerator $\left(4^{\circ} \mathrm{C}\right)$.

The solvents used in the experiments include $0.2 \mathrm{~mol} / \mathrm{L} \mathrm{Na} \mathrm{HPO}_{4}$ buffer, $0.2 \mathrm{~mol} / \mathrm{L} \mathrm{NaH} \mathrm{NO}_{4}$ buffer, $0.1 \mathrm{~mol} / \mathrm{L} \mathrm{NaOH}$ buffer, $0.2 \mathrm{~mol} / \mathrm{L} \mathrm{NaOH}$ buffer and $\mathrm{KH}_{2} \mathrm{PO}_{4}$ buffer. $0.2 \mathrm{~mol} / \mathrm{L} \mathrm{Na}_{2} \mathrm{HPO}_{4}$ buffer was compounded by putting $28.4 \mathrm{~g}$ sodium phosphte dibasic anhydrous $\left(\mathrm{Na}_{2} \mathrm{HPO}_{4}\right.$, Tianjin guangfu chemical research institute, China) into $1000 \mathrm{~mL}$ UPW. $\mathrm{KH}_{2} \mathrm{PO}_{4}-\mathrm{NaOH}$ mixed buffer of $\mathrm{pH}$ 7.4 was compounded by putting $1.36 \mathrm{~g}$ potassium dihydrogen phosphate $\left(\mathrm{KH}_{2} \mathrm{PO}_{4}\right.$, Tianjin fuchen chemical reagents factory, China) and $79 \mathrm{ml} \mathrm{NaOH}$ buffer of $0.1 \mathrm{~mol} / \mathrm{L}$ into $200 \mathrm{~mL} \mathrm{UPW}$.

3D fluorescence intensity measurements were carried out on an F-7000 FL spectrophotometer (Hitachi High-Technologies Corporation, Japan).

\section{Instrument Settings and Experiment Procedure}

$\mathrm{KH}_{2} \mathrm{PO}_{4}-\mathrm{NaOH}$ Buffers, $\mathrm{Na}_{2} \mathrm{HPO}_{4}$ or $\mathrm{NaH}_{2} \mathrm{PO}_{4}$ with different $\mathrm{pH}$ values and mother liquids of different volumes were injected into the $10 \mathrm{ml}$ test tubes, and diluted with purified water to form the working liquids and background liquids.

For the fluorescence EEM measurements of L-Tryptophan, L-Tyrosine and L-Phenylalanine, the spectrophotometer excitation wavelength ranged from $200.0 \mathrm{~nm}$ to $400.0 \mathrm{~nm}$, emission wavelength ranged from $210.0 \mathrm{~nm}$ to $500.0 \mathrm{~nm}$. Scan speed was set at $12000 \mathrm{~nm} / \mathrm{min}$ with excitation and emission sampling interval of $2.0 \mathrm{~nm}$, excitation and emission slit of $10.0 \mathrm{~nm}$, the PMT voltage was set at $600 \mathrm{~V}$. All experiments were performed at room temperature at $25^{\circ} \mathrm{C}$.

The $1^{\text {st }}$ level and $2^{\text {nd }}$ level Rayleigh scattering, Raman scattering and other background components within the fluorescence signals were corrected for the following analysis.

\section{Intrinsic Fluorescence EEM Characteristics of Tryptophan, Tyrosine and Phenyloalanine}

For tryptophan whose fluorescence quantum yield is about 0.14 , there are two strong fluorescence areas, whose center locate at $\lambda \mathrm{ex} / \lambda \mathrm{em}=230 / 350 \mathrm{~nm}, 280 / 350 \mathrm{~nm}$ respectively, and the emission wavelength ranges from about $270 \mathrm{~nm}$ to $470 \mathrm{~nm}$, as shown in Figure 1 . The fluorescence intensity excited by $280 \mathrm{~nm}$ excitation wavelength is much stronger than that by $230 \mathrm{~nm}$ excitation wavelength, and the ratio of fluorescence intensity is 1:1.10 approximately. 


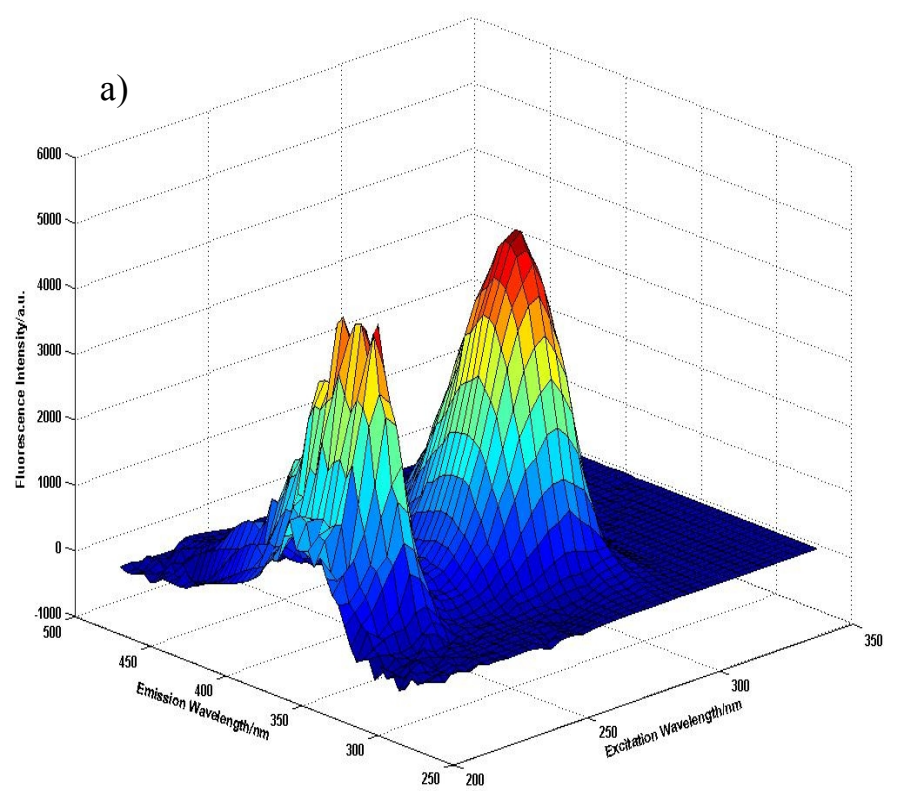

b)

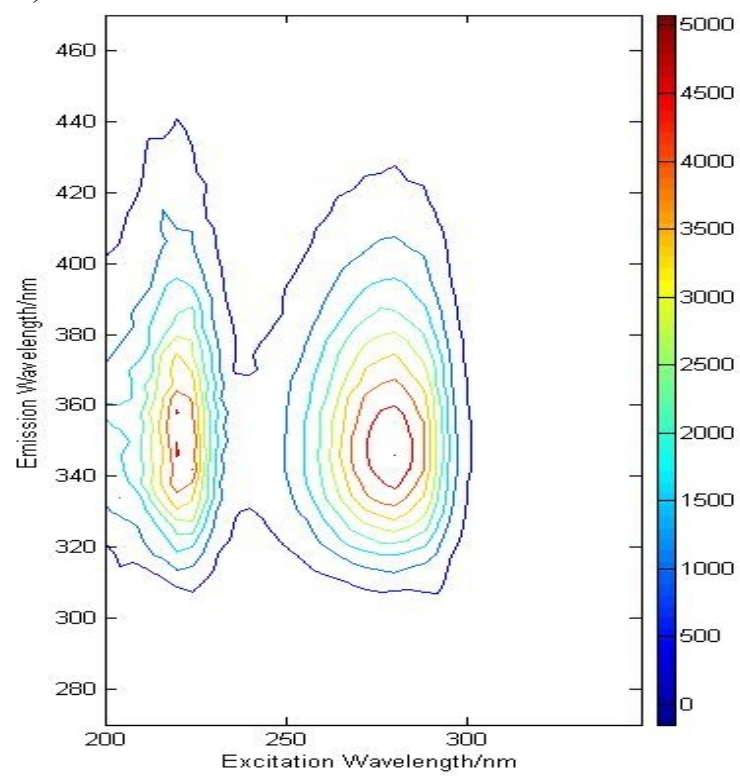

Figure1. Fluorescence intensity distribution of tryptophan@80ug/L

Tyrosine has three strong fluorescence areas, whose center locate at $\lambda \mathrm{ex} / \lambda \mathrm{em}=202 / 304 \mathrm{~nm}$, $\lambda \mathrm{ex} / \lambda \mathrm{em}=220 / 304 \mathrm{~nm}$ and $\lambda \mathrm{ex} / \lambda \mathrm{em}=274 / 304 \mathrm{~nm}$, fluorescence intensity at the positions of $\lambda$ ex $/ \lambda \mathrm{em}=202 / 304 \mathrm{~nm}$ and $\lambda$ ex $/ \lambda \mathrm{em}=220 / 304 \mathrm{~nm}$ are stronger than that of its main peak at $\lambda \mathrm{ex} / \lambda \mathrm{em}=274 / 304 \mathrm{~nm}$, as shown in Figure 2. 

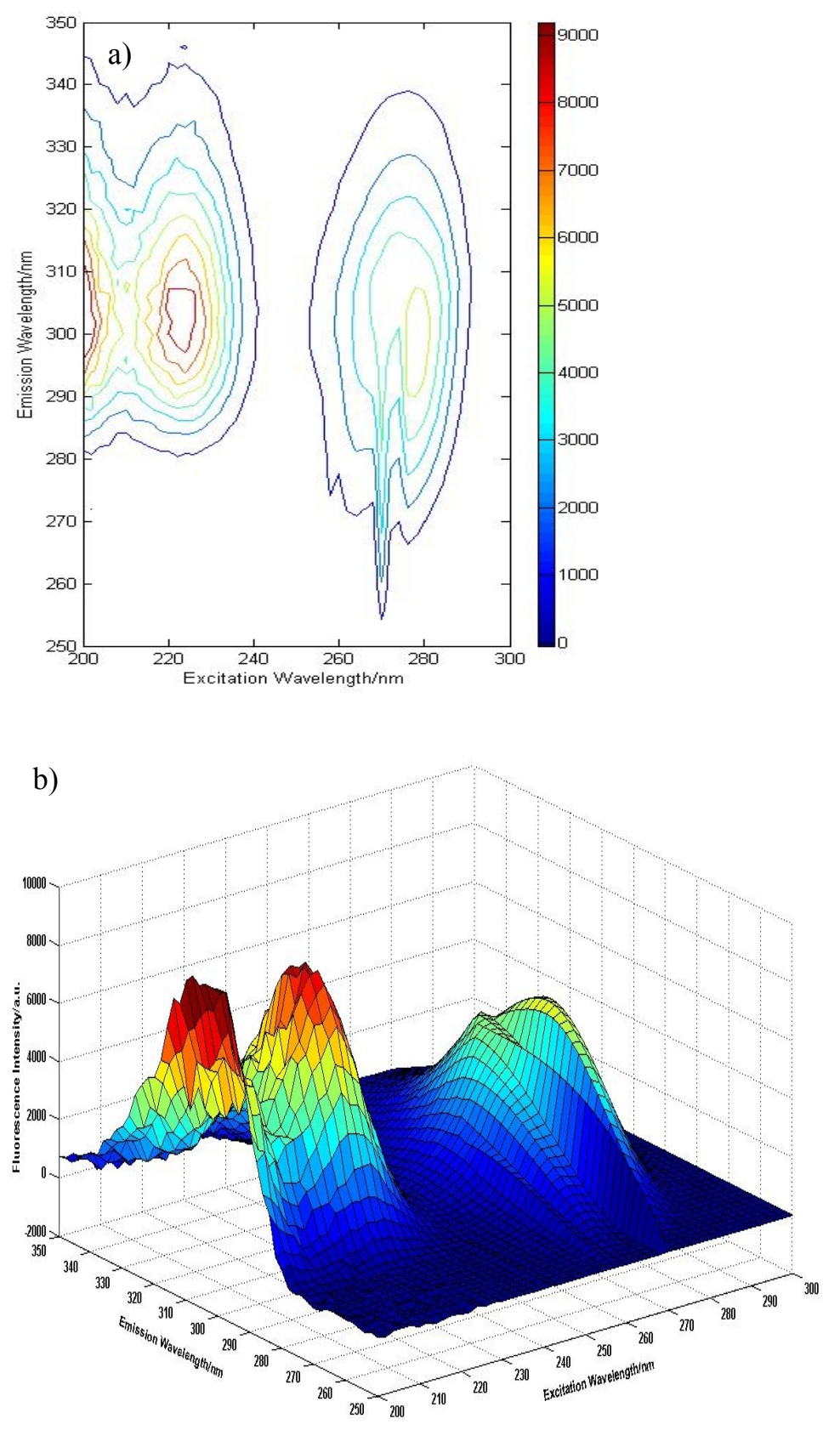

Figure2. Fluorescence intensity distribution of tyrosine@30ug/L 
a)

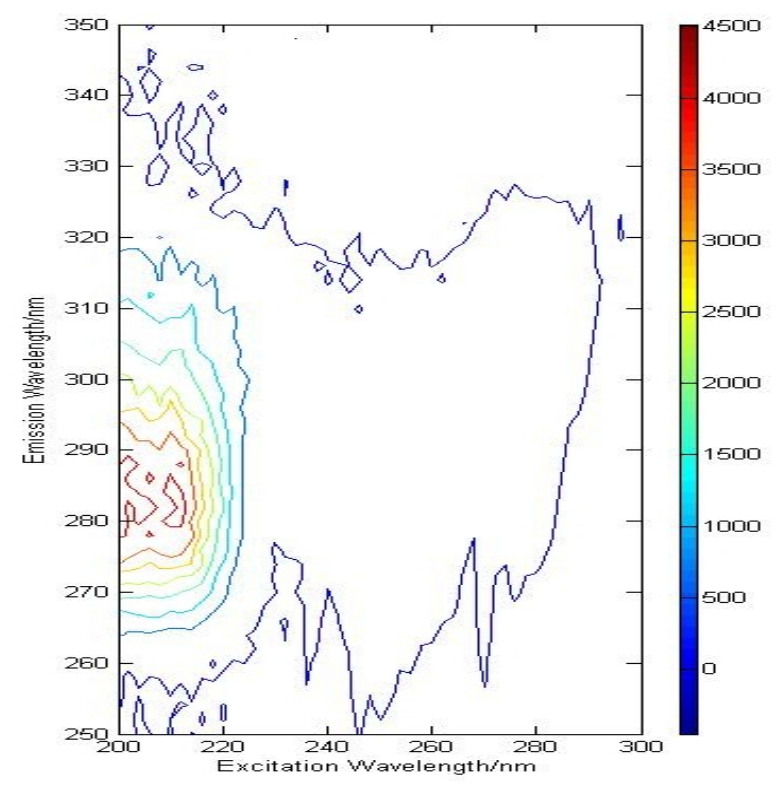

b)

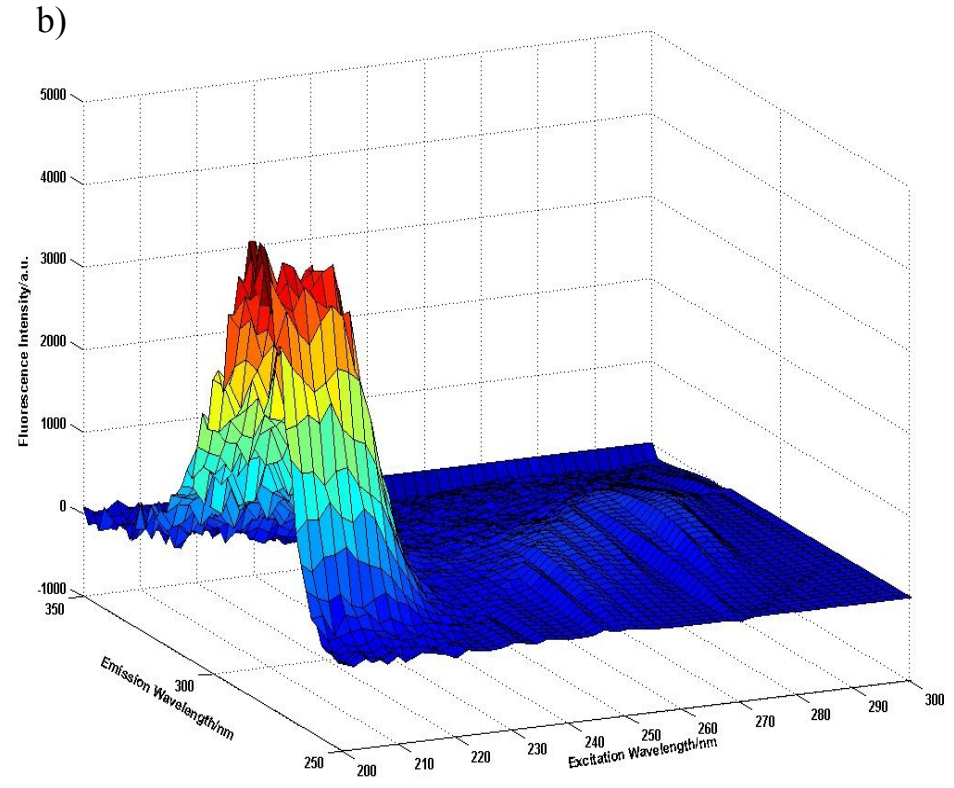

Figure3. Fluorescence intensity distribution of phenyloalanine @1350ug/L

And for phenyloalanine, there are two excitation wavelengths, the shorter one locates at $210 \mathrm{~nm}$ and the longer one locates at $260 \mathrm{~nm}$. The fluorescence of the first peak at $210 / 280 \mathrm{~nm}$ is much stronger than the second peak at $260 / 280 \mathrm{~nm}$, as shown in figure 3 .

From figure 1 to 3 , it can be conclude that, the excitation/emission wavelength of tryptophan, tyrosine and phenyloalanine locate at $\lambda \mathrm{ex} / \lambda \mathrm{em}=280 / 350 \mathrm{~nm}, 275 \mathrm{~nm} / 303$ and $260 / 280 \mathrm{~nm}$ respectively, the overlapping of these three $3 \mathrm{D}$ fluorescence spectra is obvious, as shown in figure 4. 

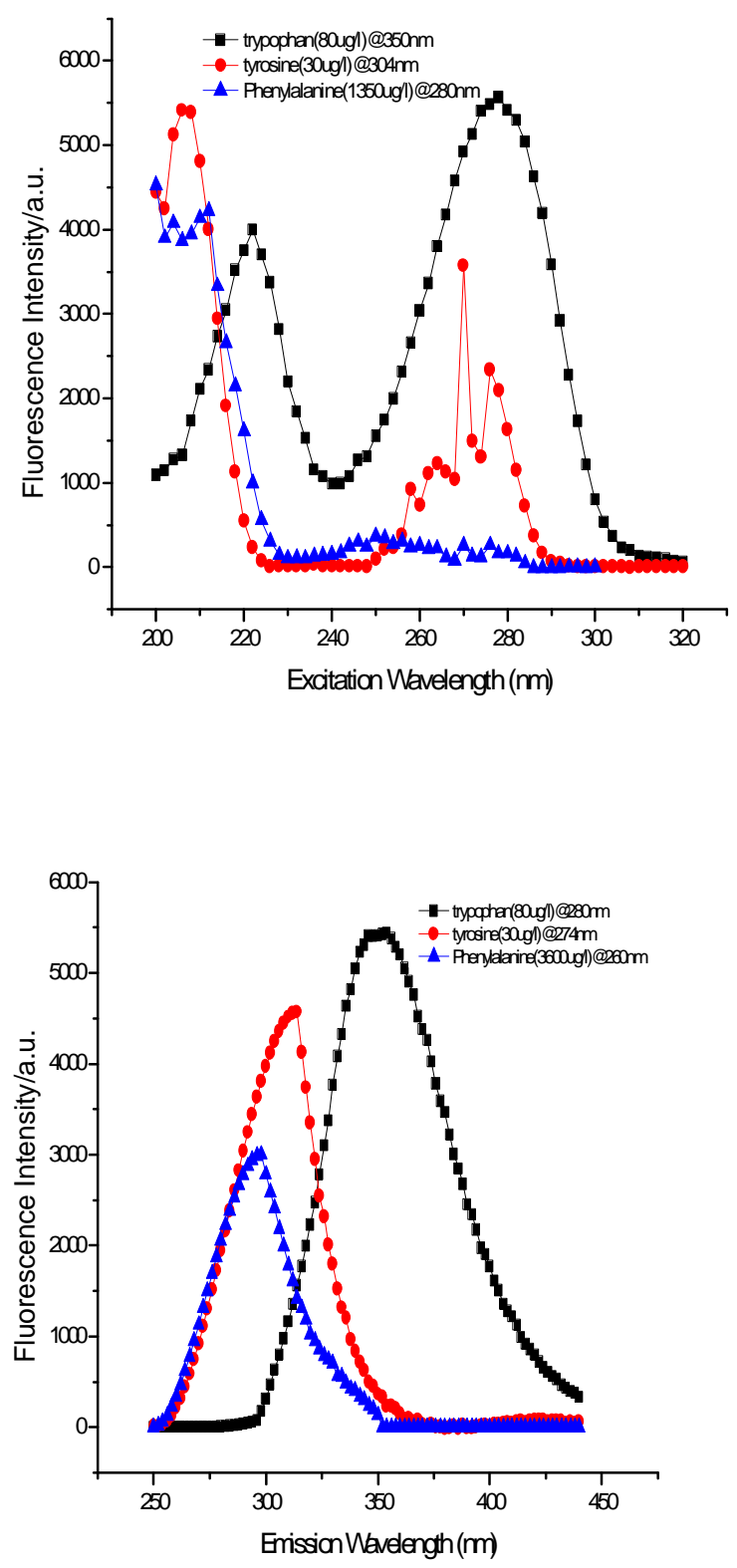

Figure4. Excitation and emission spectra of tryptophan, tyrosine and phenyloalanine @1350ug/L

\section{Linear Relationship of Fluorescence Intensity to Concentration}

According to the Lambert-Beer law, when a monochromatic light beam passes through a homogeneous, non-scattering material, the optical energy will be absorbed much stronger with higher concentration of the resolution. So for the material capable of fluorescence emission, fluorescence intensity rises with higher concentration of the resolution, the fluorescence intensity is linear to the concentration of the material resolution. The emission spectra of tryptophan at $\lambda e x=280 \mathrm{~nm}$ and the linear relationship of fluorescence intensity to resolution concentration are shown in figure5(a) and (b), the emission spectra of tyrosine at $\lambda e x=274 \mathrm{~nm}$ and the linear relationship of fluorescence intensity to resolution concentration are shown in figure5(c) and (d), and the emission spectra of phenyloalanine at $\lambda e x=260 \mathrm{~nm}$ and the linear relationship of fluorescence intensity to resolution concentration are shown in figure5(e) and (f). 

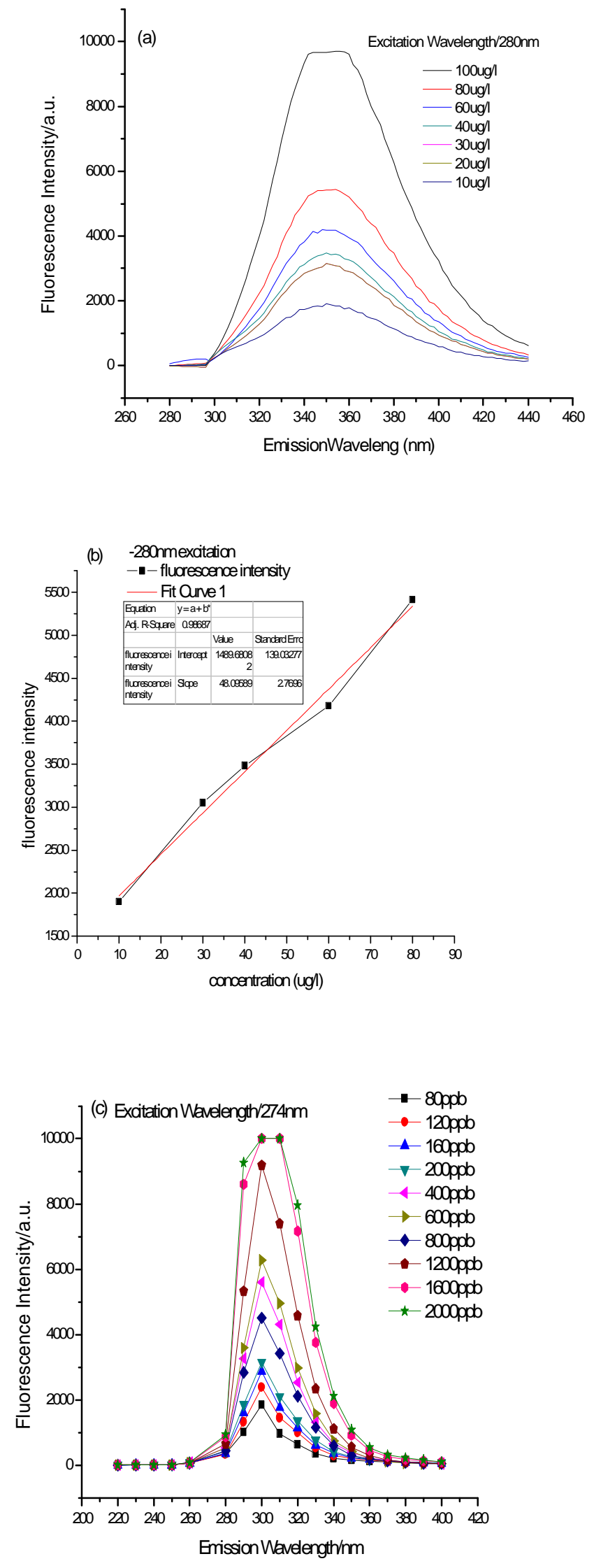

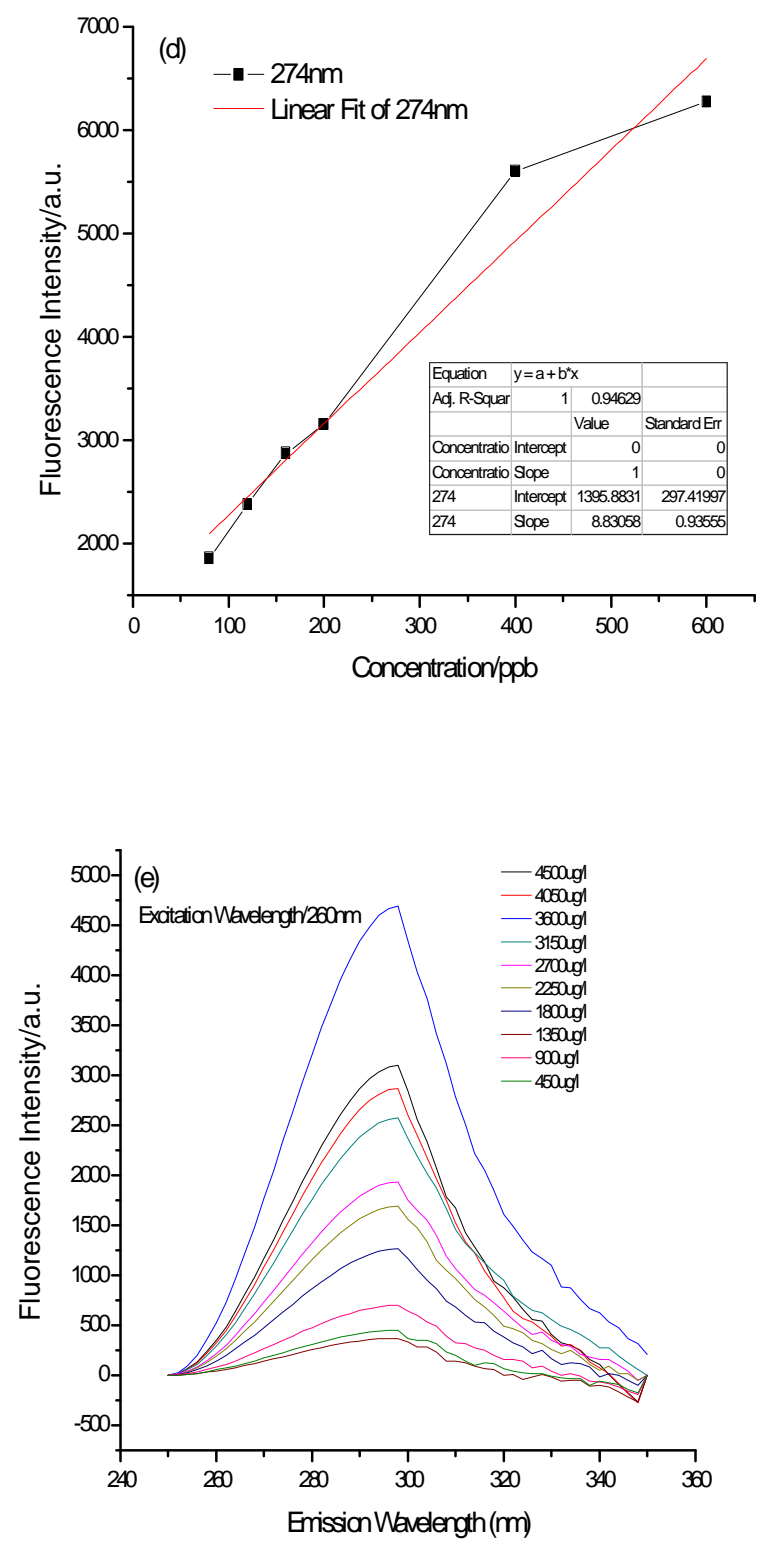


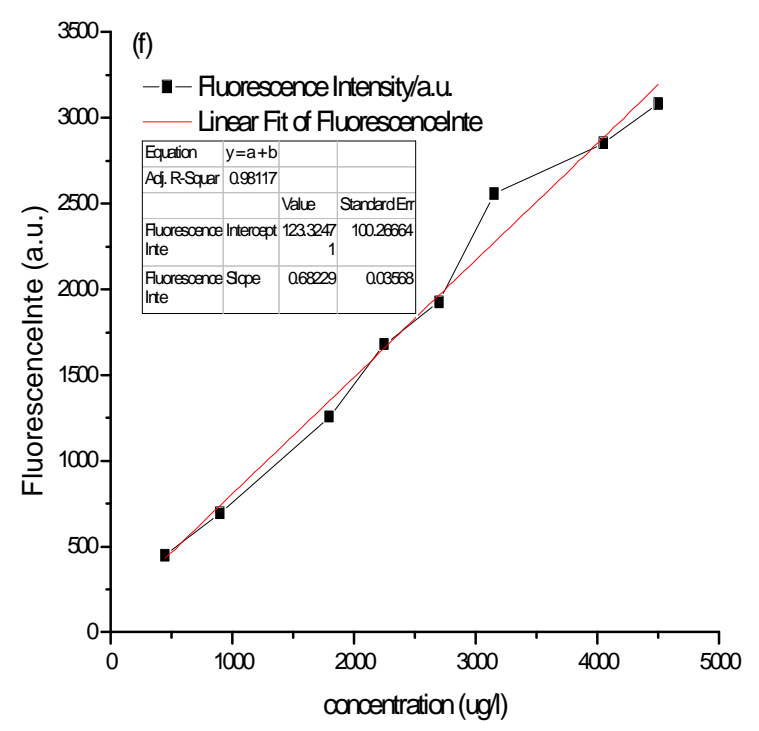

Figure5. Fluorescence spectra of tryptophan, tyrosine and phenyloalanine in different concentration at $\lambda e x=280 \mathrm{~nm}, \lambda e x=274 \mathrm{~nm}$ and $\lambda e x=260 \mathrm{~nm}$ respectively

From figure 5, the linear regression equation were obtained. the linear correlation coefficient of tryptophan comes up to 0.98687 , the fluorescence intensity is linear to the concentration of solution when the concentration is below $100 \mathrm{ug} / \mathrm{L}$. for phenyloalanine, the linear correlation coefficient is up to 0.98117 when the concentration is below $4500 \mathrm{ug} / \mathrm{L}$, and for tyrosine, the linear correlation coefficient declines to 0.94629 when the the concentration is below $600 \mathrm{ug} / \mathrm{L}$.

\section{Affection of pH to the Fluorescence Emission Intensity}

The $\mathrm{pH}$ affects the fluorescence emission intensities of the three components, especially the tryptophan and tyrosine. The fluorescence emission intensities of tryptophan and tyrosine will be strongest when the $\mathrm{pH}$ ranges from 6.5 7.5 and will decline sharply when $\mathrm{pH}$ is greater than 7.5, as shown in figure6. 


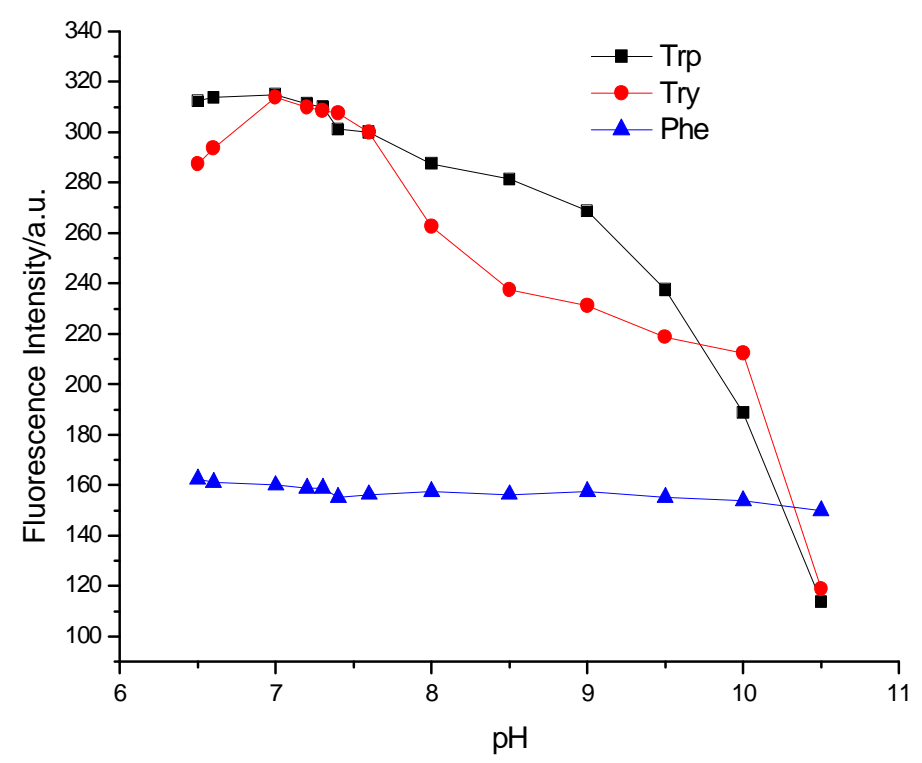

Figure6. Effect of pH/acidity of solution on fluorescence intensity of tryptophan, tyrosine and phenyloalanine respectively

\section{Conclusions}

The fluorescence of a protein or bio-aerosol or bio-agent is a mixture of the fluorescence from individual aromatic residues and coenzyme. Using fluorescence Spectrophotometer, the intrinsic fluorescent characteristics of tryptophan, tyrosine and phenyloalanine were measured with solutions of different concentration and the effect of $\mathrm{pH}$ to fluorescent profile was also discussed.

\section{Acknowledgments}

The work was supported by the National Natural Science Foundation of China named "Study on Technology of Ultraviolet Laser-induced Fluorescence LIDAR for Bioagent Remote Sensing” No. 41375026.

\section{References}

[1] Hill, S. C., Mayo, M. W., and Chang, R. K.: Fluorescence of Bacteria, Pollens, and Naturally Occurring Airborne Particles: Excitation/ Emission Spectra[R]. Army Research Laboratory, 2009.

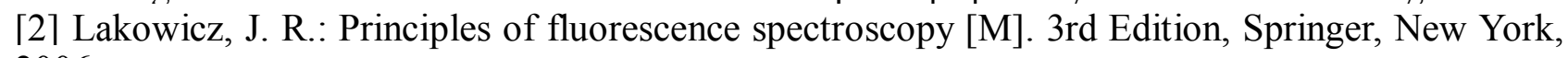
2006.

[3] Kaye, P., Stanley, W. R., Hirst, E., Foot, E. V., Baxter, K. L., and Barrington, S. Single particle multichannel bio-aerosol fluorescence sensor[J]. Opt. Express, 2005, 13: 3583-3593. 